\author{
( ) М.В. Будзинская', Д.В. Липатов², В.Г. Павлов ${ }^{1 *}$, Д.В. Петрачков
}

${ }^{1}$ Научно-исследовательский институт глазных болезней, Москва

${ }^{2}$ Национальный медицинский исследовательский центр эндокринологии, Москва

В статье проведен анализ современных публикаций по теме актуального в настоящее время направления биомедицины - исследования биомаркеров диабетической ретинопатии. Актуальность исследования обусловлена тем фактом, что выявление и идентификация биомаркеров каких-либо патологических процессов важны для скрининга, диагностики, мониторинга, профилактики и прогнозирования клинического ответа пациентов на проводимое лечение. Все это позволяет повысить эффективность и безопасность применения различных вариантов терапии, а следовательно, и улучшить отдаленный прогноз у пациентов с данным заболеванием.

В обзоре рассматриваются две основные группы биомаркеров (молекулярные и визуализируемые). Кроме того, приводится и анализируется современное состояние проблемы, рассматриваются перспективы изучения биомаркеров в контексте лечения диабетической ретинопатии. Очевидно, что поиск новых биомаркеров диабетической ретинопатии представляет важную и актуальную задачу не только для современной диабетологии, но и для эндокринологии в целом. Решение этого вопроса позволило бы перейти к выработке индивидуальных схем лечения этой патологии у пациентов с сахарным диабетом. Более тесное взаимодействие между офтальмологами и эндокринологами при лечении диабетической ретинопатии у пациентов привело бы к развитию персонализированной медицины в междисциплинарном аспекте.

КЛЮЧЕВЫЕ СЛОВА: биомаркеры; сахарный диабет; диабетическая ретинопатия; диабетический макулярный отек; персонализированная медицина

\title{
BIOMARKERS FOR DIABETIC RETINOPATHY
}

(c) Maria V. Budzinskaya', Dmitriy V. Lipatov², Vladislav G. Pavlov*, Denis V. Petrachkov

${ }^{1}$ Research institute of eye diseases, Moscow, Russia

${ }^{2}$ Endocrinology research centre, Moscow, Russia

A data analysis on the actual direction of biomedicine, the study of biomarkers in diabetic retinopathy (DR), was done. Biomarkers identification is important for screening, diagnosis, monitoring, prevention and prediction of the clinical response of the patient to the treatment. In addition, studying the biomarkers allows increase of the effectiveness and safety of using various treatment options. The review examines two main groups of biomarkers, molecular and visualised, which shows the current state of the problem and the prospects for studying biomarkers in the context of the DR treatment. Nowadays, searching for and finding new biomarkers is important and will allow us to develop individual treatment regimens for DR and personalised medicine in an interdisciplinary aspect: ophthalmology and endocrinology.

KEYWORDS: biomarkers; diabetes mellitus; diabetic retinopathy; diabetic macular edema; personalized medicine

Понятие биологического маркера «биомаркер» стало широко использоваться в 80-х годах прошлого века. Это определение предшествовало термину «суррогатная конечная точка» в клинических исследованиях [1] в качестве характеристики, которая объективно измеряется и оценивается как показатель нормальных биологических, патологических процессов или фармакологических реакций на терапевтическое вмешательство [2].

В настоящее время термин биомаркер используется более широко и определяется как исследуемый параметр, измерение которого отличается высокой точностью, надежностью и воспроизводимостью, что позволяет отражать напряженность физиологических процессов, состояние здоровья, степень риска или сам факт прогрессирования заболевания, а также его стадию и прогноз [3].
Благодаря выявлению специфического набора биомаркеров, можно подобрать оптимальную стратегию ведения пациента в соответствии с задачами персонализированной медицины [4] - широкой и быстро развивающейся области здравоохранения, опирающейся на уникальную клиническую, генетическую, геномную и экологическую информацию каждого человека [5].

Пандемией XXI в. в развитых странах считается сахарный диабет (СД). Общая численность пациентов с СД в РФ неуклонно растет. Так, к концу 2016 г. она составила 4,4 млн человек (2,9\% населения РФ), из них: 92\% (4 млн) - с СД 2 типа (СД2) и 6\% (255 тыс.) - с СД 1 типа (СД1) и 2\% (75 тыс.) - с другими типами СД [6]. Соответственно, особое внимание со стороны офтальмологов и эндокринологов должно уделяться диабетической 
ретинопатии (ДР) - хроническому прогрессирующему заболеванию микрососудов сетчатки, поражающему пациентов, длительное время страдающих СД [7].

ДР является основной причиной слепоты у трудоспособного населения развитых стран и, наряду с возрастной макулярной дегенерацией (ВМД) и глаукомой, входит в число ведущих причин снижения остроты зрения в возрастной группе старше 65 лет.

В 2016 г. распространенность ДР в РФ составила при СД1 3806 на 10 тыс. (38,3\%) взрослых пациентов (показатель остается на уровне 2013 г.) и при СД2 1497 на 10 тыс. (15\%) взрослых пациентов (некоторое снижение по сравнению с уровнем 2013 г.). При этом динамика показателей заболеваемости (новые случаи ДР/год) в 2016 г. имела небольшую тенденцию к повышению по сравнению с уровнем 2013 г.: при СД1 - 187,8 и при СД2 - 114,9 на 10 тыс. взрослого населения. Структура новых случаев ДР в 2016 г. следующая: непролиферативная стадия (НПДР) СД1 - 71,4\%, СД2 - 80,3\%, препролиферативная (ППДР) - 16,4\%/13,8\%, пролиферативная (ПДР) - 12,1\%/5,8\%, терминальная - 0,2\%/0,1\% при СД1/СД2 соответственно, что свидетельствует о преимущественном выявлении ДР на ранней стадии $[8,9]$.

Диабетический макулярный отек (ДМО) может возникать на любой стадии заболевания и занимает прочные позиции в снижении остроты зрения у пациентов с СД, а частота его составляет 6,81\% из всех пациентов, страдающих СД [10].

Несмотря на стабильные показатели распространенности СД, а также достигнутое уменьшение вероятности слепоты в результате ДР [6], эффект от хирургического и терапевтического лечения ДМО значительно меньше, чем в рандомизированных клинических исследованиях (РКИ) $[11,12]$.

В свете вышеизложенного, весьма интересным и перспективным является изучение и поиск новых биомаркеров, что даст возможность верификации субклинических стадий ДР (в рамках скрининга), более точной оценки тяжести заболевания, оценки прогноза развития заболевания. Перечисленные мероприятия, в свою очередь, позволят перейти к персонализированному лечению заболевания, а именно оценке ответа на различные варианты терапии с выбором наиболее подходящего для данного пациента и мониторинга эффективности проведенного лечения.

\section{МОЛЕКУЛЯРНЫЕ БИОМАРКЕРЫ}

Молекулярные (неотображаемые) биомаркеры имеют биофизические свойства, которые возможно измерить в биологических средах (плазма и сыворотка крови, влага передней камеры, стекловидное тело) [3].

Одними из первых появились работы по изучению формулы крови и влияния системы гемостаза на развитие и течение ДР. Имеются противоречивые данные относительно гиперкоагуляции. Например, исследование D.Q. Borsey и соавт. (1984 г.) показало, что, несмотря на ожидания, гиперкоагуляция не подтвердилась [13]. Изменения гемостаза были выявлены и коррелировали с ДР: уровень бета-тромбоглобулина, тромбоцитарного фактора 4, антитромбина III, фибриногена и значения антигена, связанного с фактором VIII.
B отличие от предыдущего исследования, R. Madan и соавт. (2010 г.) предположили, что состояние гиперкоагуляции является одним из факторов развития микрососудистых осложнений СД, о чем свидетельствуют снижение фибринолиза и повышение свертываемости [14]. Кроме того, они выявили, что уровень плазменного ингибитора активации плазминогена I типа и активность фактора фон Виллебранда были значительно повышены у пациентов с СД и микрососудистыми осложнениями, а активность белка $S$ - значительно ниже. Таким образом, эти противоречия не позволяют использовать параметры гемостаза как биомаркеры при ДР.

Ряд исследователей предполагали тесную связь между уровнями липидов и ДМО при использовании этих показателей как биомаркеров более тяжелого его течения с увеличением количества твердых экссудатов, однако это не было подтверждено метаанализом, включающим только проспективные литературные источники. Несмотря на то, что уровень общего холестерина, липопротеинов низкой плотности и триглицеридов значимо выше у пациентов с ДМО, метаанализ РКИ показал, что в группе, получавшей гиполипидемические средства, не было значимого снижения риска увеличения твердых экссудатов и усиления ДМО по сравнению с группой плацебо [15].

При длительном наблюдении (24 мес) пациентов с ДМО такие системные факторы, как уровень глюкозы и гликированный гемоглобин ( $\left.\mathrm{HbA}_{1 c}\right)$ в сыворотке крови, функция почек, индекс массы тела (ИМТ) и артериальное давление не оказывали влияния на максимально корригированную остроту зрения (МКО3) [16].

Оценка прогрессирования ДР за год инсулинотерапии в зависимости от темпов изменения уровня $\mathrm{HbA}_{1 c}$ и его диапазона показала, что при сохранении исходного повышенного уровня $\mathrm{HbA}_{1 с}$ либо его дальнейшем увеличении прогрессирование ДР отмечено в 54,6\% случаев. Высокий уровень прогрессирования (44,4\%) выявлен также при снижении уровня $\mathrm{HbA}_{1 с}$ на $3 \%$ и более [17].

С начала 2000-х годов началось исследование содержания цитокинов, факторов роста и других активных веществ во влаге передней камеры и стекловидном теле. Была выявлена ведущая роль фактора роста эндотелия сосудов (VEGF - англ. Vascular endothelial growth factor) в патогенезе ДР и ДМО [18].

В настоящее время наиболее изучен VEGF-A, который существует в различных изоформах, образующихся в результате альтернативного сплайсинга единственного гена VEGF-A, содержащего 8 экзонов. Основной биологический эффект VEGF опосредуется через взаимодействие с рецептором VEGF-R ${ }_{2}$ относящимся к группе трансмембранных тирозиновых киназ [19].

VEGF необходим также для процессов эмбриогенеза и раннего постнатального ангиогенеза. У взрослых VEGF в сосудистой стенке действует на разных уровнях: как фактор, обеспечивающий выживание эндотелиальных клеток, усиливает сосудистую проницаемость и действует как мощный вазодилататор [20]. При СД действие VEGF, секретируемого клетками ретинального пигментного эпителия, проявляется в росте новообразованных сосудов и усилении отека сетчатки. Для СД1 более характерно развитие ПДР с появлением неоваскуляризации, в то время как при СД2 чаще отмечается ДМО с потерей центрального зрения. 
Исследование Борзиловой Ю.А. и соавт. (2016 г.) уровня VEGF-A в слезной жидкости у пациентов с различными стадиями ДР дало следующие результаты: уровень VEGF-A в слезе оказался значительно выше у пациентов с ДР по сравнению с пациентами, страдающими СД, но без признаков ДР. Значимых различий в уровне VEGF-A у пациентов с НПДР и ПДР не выявлено. В то же время у пациентов с тракционной отслойкой сетчатки и выраженным глиозом на фоне ПДР, но без признаков неоваскуляризации переднего отдела глаза, выявлено значительное снижение уровня VEGF-A. При этом уровень VEGF-A коррелировал с активностью неоваскулярного процесса, определенного по данным флуоресцентной ангиографии (ФАГ) [21]. Таким образом, определение уровня VEGF В стимулированной слезной жидкости в силу неинвазивности метода имеет перспективу использования в качестве биомаркера ДР.

Так как ДМО - наиболее распространенная причина снижения остроты зрения у пациентов с СД, появление анти-VEGF-препаратов диктовало поиск новых биомаркеров [22]. Ингибиторы VEGF - это моноклональные антитела, которые селективно связываются с VEGF, блокируя его действие. Они подавляют неоангиогенез в опухолях, лишая их возможности дальнейшего роста. Полученные за последнее время данные об участии VEGF в развитии ДР позволили применять анти-VEGF в качестве консервативного лечения ДР [23].

Несмотря на то что анти-VEGF-препараты являются терапией первой линии, у 50\% пациентов с ДМО все еще остается отек спустя 1 год лечения от начала лечения. В связи с этим началось активное изучение влияния воспаления на прогрессирование ДМО [24]. При изучении уровней VEGF, молекул межклеточной адгезии 1 (ICAM - англ. Intercellular adhesion molecule 1), интерлейкина 6 (IL - англ. Interleukin 6) и хемотаксического белка моноцитов (МСР англ. Monocyte chemotaxis protein) в стекловидном теле, данные показатели были значительно выше у пациентов с ДМО, чем в контрольной группе (пациенты без СД) или у пациентов с СД, но без ДР. Уровень фактора роста пигментного эпителия (PEDF - англ. Pigment epithelium-derived factor) был значительно ниже у пациентов с ДМО, чем в сравниваемых группах. VEGF, ICAM-1, IL-6, MCP-1 и PEDF значимо коррелировали с толщиной сетчатки в центральной зоне глазного дна. Также было выявлено, что VEGF и ICAM-1 оказывали более сильное влияние на тяжесть течения ДМО, чем другие изученные факторы [25].

Результаты этих исследований могут быть полезны для выяснения механизма нарушения гематоэнцефалического барьера у пациентов с ДМО и для разработки новых методов лечения. Однако это было перекрестное исследование, и размер выборки был относительно небольшим, поэтому потребуется дальнейшее исследование для подтверждения корреляционной связи между факторами воспаления стекловидного тела и проницаемостью сосудов [25].

Отсутствие эффекта от лечения анти-VEGF-препаратами может объясняться наличием других веществ, способствующих прогрессированию независимо от VEGF, и именно их выявление и использование в качестве биомаркеров прогрессирования заболевания могут стать шагом к персонализированной терапии. ANGPTL4 (angiopoietin-like 4) является цитокином, отвечающим за прогрессирование ДР и ДМО независимо от VEGF. При ишемии внутренних слоев сетчатки повышается экспрессия этого белка, и он может быть потенциальной мишенью при лечении ДМО и биомаркером индуцированной ишемической ретинопатии, включая ДР [26].

На основании исследований, изучавших молекулярные биомаркеры, были сделаны выводы, что устойчивая гипергликемия приводит к окислительному стрессу и эпигенетические модификации индуцируют воспаление, что приводит к повышению провоспалительных цитокинов и хемокинов [25]. В дальнейшем в широкую клиническую практику вошло применение кортикостероидов при ДМО и появление новых препаратов - интегринов, влияющих на окислительный стресс [27].

Большим вопросом остается определение биомаркеров, приводящих к развитию фиброваскулярных мембран при ДР, связанных с экстраретинальным неоангиогенезом. Обнаружены цитокины, индуцирующие образование фиброза, - трансформирующий фактор роста $\beta$, PDGF и фактор роста соединительной ткани. Под их воздействием происходят трансдифференцировка, пролиферация и миграция миофибробластов и запускаются механизмы продукции внеклеточного матрикса, обладающего способностью к сокращению. В экспериментальных работах на мышах продемонстрирована возможность трансдифференцировки перицитов и периваскулярных мезенхимальных клеток в миофибробласты в различных тканях и органах. Тем не менее источник миофибробластов сетчатки человека остается неизвестным [28, 29]. Учитывая быстрое развитие или прогрессирование тракционных процессов на глазном дне после инъекций анти-VEGF-препаратов, можно предположить, что оставшиеся перициты после абляции эндотелиальных клеток новообразованных сосудов и являются источником миофибробластов, однако данная теория требует дальнейшего изучения молекулярных биомаркеров при пролиферативной стадии заболевания [30].

Исследование нейротрофического фактора мозга (BDNF - англ. Brain-derived neurotrophic factor) во влаге передней камере и в сыворотке крови выявило его снижение до начала клинического развития ДР [31].

Альбуминурия была независимым фактором риска потери слоя нервных волокон у пациентов с СД2 без почечной недостаточности. Может потребоваться тщательное обследование диска зрительного нерва, особенно у пациентов с СД2 и альбуминурией [32].

Несмотря на большое количество исследований, проведенных на эту тему, нет четкой взаимосвязи между молекулярными биомаркерами и клиническими проявлениями, прогнозом течения и прогнозом ответа на лечение ДР. Изучение данной проблемы в перспективе позволит установить корреляцию между изменениями в биологических образцах и визуально наблюдаемыми биомаркерами на сетчатке.

\section{ОТОБРАЖАЕМЫЕ БИОМАРКЕРЫ}

Отображаемые (визуализируемые) биомаркеры это биомаркеры, определяемые на изображениях, полученных при помощи визуализирующих методик, таких как фоторегистрация изменений глазного дна, включающая ФАГ, оптическую когерентную томографию (ОКТ) 
и ОКТ в ангиорежиме (ОКТ-А). Перспективным может быть мультимодальный подход к идентификации биомаркеров, полученных при помощи перечисленных методов обследования [33].

Наиболее известными биомаркерами, полученными при фоторегистрации глазного дна (7 центральных полей) в исследовании ETDRS (от англ. The Early treatment diabetic retinopathy study), являются признаки, служащие достаточно четкими показателями прогрессирования ДР и позволяющие точно установить стадию заболевания: микроаневризмы, ретинальные геморрагии, «четкообразные» вены, новообразованные сосуды [34].

Однако стандартные методы не позволяют выявлять изменения в периферических отделах сетчатки, которые могут иметь решающее значение в прогрессировании ДР. Например, у пациентов с периферическим повреждением сетчатки вероятность перехода в пролиферативную стадию ДР выше в 4,7 раза [35]. Эти результаты показывают, что детальное исследование периферии сетчатки предоставляет важную информацию, необходимую для полной оценки риска прогрессирования ДР. Таким образом, наличие периферических изменений может быть биомаркером прогрессирования заболевания.

Фотографирование глазного дна с помощью $200^{\circ}$ широкопольной камеры и фоторегистрация по ETDRS показали, что диабетические поражения сетчатки возникают на ее периферии за пределами полей ETDRS у 40\% глаз и что эти поражения могут быть оценены как более тяжелые в 12,7\% глаз по сравнению с оценкой тяжести, полученной на основании исследования 7 полей по ETDRS [37].

Наличие твердых экссудатов является предиктором субретинального фиброза и снижения зрения при ДМО [38], соответственно, может быть использовано как биомаркер прогноза лечения.

Биомаркеры, полученные на основе фоторегистрации, широко используются в настоящее время для установки стадии заболевания и определения показаний для некоторых видов лечения (панретинальная лазеркоагуляция). Однако стандартная фоторегистрация (7 центральных полей по ETDRS) не позволяет выявить изменения на периферии сетчатки, что требует использования нового оборудования. Кроме того, фоторегистрация не отражает гемодинамических нарушений, в большинстве случаев встречающихся при ДР.

Современные фундус-камеры имеют возможность исследовать поле до $200^{\circ}$, даже без мидриаза, что позволяет осуществлять осмотр более чем $80 \%$ от общей поверхности сетчатки. Тем не менее широкопольная визуализация имеет некоторые ограничения, в том числе искажение изображений из-за сферической природы глазного яблока, ложной цветопередачи, а также высоких затрат на оборудование. Из-за этих ограничений традиционная $30^{\circ}$ фотография глазного дна продолжает оставаться стандартным методом, используемым для получения его изображений [36].

ОКТ-биомаркеры, такие как центральная толщина сетчатки (ЦТС) [39, 40], дезорганизация внутренних слоев сетчатки, DRIL (от англ. Disorganization of retinal inner layers) $[40,41]$, и разрушение эллипсоидальной зоны, EZ (от англ. Ellipsoid zone) [42], наличие субретинальной жидкости (СРЖ) могут коррелировать с максимальной коррекцией остроты зрения (МКО3) после терапии анти-VEGF [42].
Уменьшение жидкости в наружных слоях сетчатки является более надежным биомаркером восстановления MKO3, чем ЦTC, DRIL и дезорганизация EZ, по данным OKT после лечения ДМО анти-VEGF-препаратами [43].

DRIL является биомаркером плохого прогноза по зрению после резорбции ДМО, предполагается, что ишемия при ДР с потерей нормальной васкуляризации сетчатки и расширением фовеолярной аваскулярной зоны (ФАЗ) способствуют формированию DRIL [44].

Однако ОКТ-биомаркеры являются не до конца изученными, поэтому идет поиск других биомаркеров, позволяющих оценить прогноз лечения ДМО.

Толщина хориоидеи отличается у пациентов с пролиферативной стадией ДР, также отмечено значимое уменьшение хориоидеи у пациентов с ДМО [45]. У пациентов с СД1 при длительности заболевания более 10 лет выявляются изменения толщины хориоидеи в центральной зоне, и эти изменения происходят раньше возникновения ДР [46]. После проведения панретинальной лазерной коагуляции (ПРЛК) значимо уменьшается толщина макулярной и перипапиллярной хориоидеи [47]. Таким образом, толщина хориоидеи может быть кандидатом на роль биомаркера раннего выявления ДР, однако это требует дальнейшего изучения.

Гиперрефлективные точки сетчатки (HRS - англ. Hyperreflective retinal spots) описаны при разных хориоретинальных сосудистых и дегенеративных заболеваниях. HRS - это мелкие, иногда точечные гиперрефлективные зоны, видимые на В-сканах ОКТ как во внутренних, так и наружных слоях сетчатки [48]. Имеются разные взгляды на природу этого биомаркера. Если HRS $\leq 30$ мкм (с такой же отражательной способностью, как у слоя нервных волокон, и отсутствием тени), то они представляют собой активированные клетки микроглии; при HRS >30 мкм (отражательная способность такая же, как комплекс ретинального пигментного эпителия (РПЭ) - мембрана Бруха с наличием тени и расположение в наружных слоях сетчатки) - то это твердый экссудат; при HRS > 30 мкм (с наличием тени и расположением во внутренних слоях сетчатки) представляют собой микроаневризмы [49].

Однако ряд исследований говорит об ином происхождении HRS. Гистологические исследования кадаверных глаз человека показывают, что HRS - это мигрировавшие клетки ретинального пигментного эпителия (РПЭ), которые проникают вплоть до наружного плексиформного слоя, часто сочетаются с нарушениями наружной пограничной мембраны сетчатки и/или эллипсоидной зоны [50]. В других исследованиях было показано, что HRS могут быть как клетками РПЭ, так и клетками иного происхождения, заполненными липидами. Несмотря на растущее число опубликованных статей об оценке HRS при ДР, по-прежнему отсутствует консенсус в отношении их происхождения, местоположения, характеристик и значимости [50, 51, 52].

Витреомакулярная адгезия (BMA) - биомаркер хорошего потенциала для терапии ангиогенными препаратами ДМО. Разницы между широкой и узкой адгезией не выявлено [53].

Биомаркеры, идентифицированные с помощью однофакторного анализа как предикторы прогрессирования до ПДР, следующие: ЦТС, объем сетчатки в центральной зоне, фокальный/диффузный отек, наличие СРЖ, двусторонний ДМО, МКОЗ до лечения, внутриглазное давление, 
стадия ДР, контрастная чувствительность, очаговая/диффузная неперфузия [54].

При исследовании эффективности лечения ДМО при помощи импланта дексаметазона было выявлено, что наличие СРЖ, отсутствие HRS и непрерывность EZ являются предикторами хорошего ответа на лечение [55].

Изменения, выявленные на ОКТ, также могут быть биомаркерами других осложнений СД: уменьшение слоя нервных волокон у пациентов с СД значимо связано с наличием периферической диабетической нейропатии [56].

В настоящее время ОКТ-биомаркеры находятся в фокусе изучения учеными-офтальмологами. Этому способствуют неинвазивность и быстрота метода ОКТ. Однако до сих пор нет ответа на вопрос, какие ОКТ-биомаркеры имеют достаточную специфичность и воспроизводимость для прогнозирования ответа на различные варианты терапии.

ФАГ также существует в широкопольном варианте и позволяет выявить такой биомаркер, как зоны ишемии наряду с новообразованными сосудами и зонами просачивания. Все эти биомаркеры используются для оценки тяжести, стадии и показаний к началу лечения. Определенными минусами ФАГ являются его инвазивность и отсутствие зарегистрированной коммерческой формы флуоресцеина в РФ на момент написания данного обзора.

В последнее время стало доступно использование В широкой практике ОКТ-А, за счет неинвазивности которого можно оценить как поздние, так и ранние изменения микроциркуляторного русла.

ОКТ-А - современный информативный метод, позволяющий выявлять ряд изменений в гемодинамике сетчатки, которые служат биомаркерами ДР. Например, имеется корреляция между прогнозом по МКОЗ и площадью ФАЗ. Также ОКТ-А позволяет определять капиллярную плотность поверхностного, срединного и глубокого капиллярных сплетений [57].

В отношении ОКТ-А также ведутся исследования широкопольного применения (с созданием ОКТ-А карты до $200^{\circ}$ ). Выявлена связь между площадью неперфузии и стадией ДР: в глазах без ДР площадь неперфузии составляет $-0,1 \%$, с НПДР - 2,1\%, с ПДР - 8,5\% [58].

ОКТ-А позволяет получить количественное измерение следующих биомаркеров ОСТ-А: извилистость кровеносных сосудов, калибр сосудов крови, индекс периметра сосудов, плотность кровеносных сосудов, площадь ФАЗ и неровности контура ФАЗ. Плотность кровеносных сосудов в височно-периферической области показывает лучшую чувствительность к раннему выявлению ДР. Комбинированные признаки, то есть объединение всех количественных признаков ОСТ-А, демонстрируют по- вышение точности классификации ДР [59].

Перспективным является исследование ФАЗ, капиллярной плотности и других изменений в поверхностном и глубоком капиллярных сплетениях (SCP - англ. superficial capillary plexuses и DCP англ. - deep capillary plexuses) по ОКT-A при СД, как это было проведено Lee J. и соавт. (2016 г.), результаты исследований которых показали, что пациенты с СД без клинических проявлений ДР показали более низкую капиллярную плотность и больший размер ФАЗ в поверхностном и глубоком капиллярных сплетениях сетчатки.

Отображаемые биомаркеры могут служить для прогнозирования эффективности анти-VEGF-терапии. Плохой ответ на анти-VEGF-терапию можно прогнозировать при большом количестве микроаневризм, большой площади фовеальной аваскулярной зоны и сниженной плотности кровотока в глубоком ретинальном сплетении. Полученные данные коррелировали с нарушением целостности наружного плексиформного слоя и низкими зрительными функциями [60].

Таким образом, ОКТ-А позволяет получить биомаркеры, отражающие степень повреждения капиллярного русла сетчатки, и прогнозировать ответ на терапию антиангиогенными препаратами.

\section{ЗАКЛЮЧЕНИЕ}

Результаты анализа доступной литературы подтверждают установленный факт важности исследования биомаркеров ДР у пациентов с СД. Изучение биомаркеров позволяет повысить эффективность и безопасность применения различных вариантов терапии ДР, расширить знания патофизиологии ДР. Именно применение биомаркеров в клинической практике лежит в основе патогенетического лечения ДР, что дает возможность обеспечить преемственность лечения данной категории пациентов. Однако, несмотря на многочисленные исследования, ряд вопросов в настоящее время остается дискутабельным и открытым для обсуждения.

\section{ДОПОЛНИТЕЛЬНАЯ ИНФОРМАЦИЯ}

Конфликт интересов. Авторы декларируют отсутствие явных и потенциальных конфликтов интересов, связанных с публикацией настоящей статьи.

Участие авторов. Липатов Д.В., Будзинская М.В. - подбор материала; Павлов В.Г. - систематизация материала; Петрачков Д.В. редактирование материала. Все авторы внесли значимый вклад в подготовку статьи, прочли и одобрили финальную версию перед публикацией.

\section{СПИСОК ЛИТЕРАТУРЫ | REFERENCES}

1. Aronson JK. Biomarkers and surrogate endpoints. Br J Clin Pharm. 2005;59(5):491-494. doi: https://doi.org/10.1111/J.1365-2125.2005.02435.X

2. Biomarkers Definitions Working Group. Biomarkers and surrogate endpoints: preferred definitions and conceptual framework. Clin Pharm Ther. 2001;69(3):89-95. doi: https://doi.org/10.1067/Mcp.2001.113989

3. Мирошниченко И.И., Птицина С.Н. Биомаркеры в современной медико-биологической практике // Биомедицинская химия. -
2009. - T. 55. - №4. - C. 425-440. [Miroshnichenko II, Ptitsyna $\mathrm{SN}$. Biomarkers in the modern medical and biologic practice. Biomed khim. 2009;55(4):425-440. (In Russ.)]

4. Waldman S, Terzic A. Targeted diagnostics and therapeutics for individualized patient management. Biomark Med. 2007;1 (1):3-8. doi: https://doi.org/10.2217/17520363.1.1.3

5. Ginsburg GS, Willard HF. Genomic and personalized medicine: foundations and applications. Trans/ Res. 2009;154(6):277-287. doi: https://doi.org/10.1016/J.Trsl.2009.09.005 
6. Дедов И.И., Шестакова М.В., Викулова О.К. Эпидемиология сахарного диабета в Российской Федерации: клиникостатистический анализ по данным Федерального регистра сахарного диабета // Сахарный диабет. - 2017. - T. 20. №1. - C.13-41. [Dedov II, Shestakova MV, Vikulova OK. Epidemiology of diabetes mellitus in Russian Federation: clinical and statistical report according to the Federal diabetes registry. Diabetes mellitus. 2017;20(1):13-41. (In Russ.)] doi: https://doi.org/10.14341/Dm8664

7. Федеральные клинические рекомендации: диагностика и лечение диабетической ретинопатии и диабетического макулярного отека. - M., 2013. [Federal clinical guidelines: diagnosis and treatment of diabetic retinopathy and diabetic macular edema. Moscow; 2013. (In Russ.)] Доступно по: https://zodorov.ru/ federalenie-klinicheskie-rekomendacii-diagnostika-i-lechenie-d.html. Ссылка активна на 12.11.2019.

8. Липатов Д.В., Викулова О.К., Железнякова А.В., и др. Эпидемиология диабетической ретинопатии в Российской Федерации по данным Федерального регистра больных сахарным диабетом (20132016 гг.) // Сахарный диабет. - 2018. - T. 21. — №4. - C. 230-240. [Lipatov DV, Vikulova OK, Zheleznyakova AV, et al. Trends in the epidemiology of diabetic retinopathy in Russian Federation according to the Federal diabetes register (2013-2016). Diabetes mellitus. 2018;21 (4):230-240. (In Russ.)] doi: https://doi.org/10.14341/Dm9797

9. Дедов И.И., Шестакова М.В., Майоров А.Ю., и др. Алгоритмы специализированной медицинской помощи больным сахарным диабетом / Под ред. И.И. Дедова, М.В. Шестаковой, А.Ю. Майорова. 9-й вып // Сахарный диабет. - 2019. - Т. 22. — №1S1. C. 1-144. [Dedov II, Shestakova MV, Mayorov AYu, et al. Standards of specialized diabetes care. Ed. by Dedov II, Shestakova MV, Mayorov AYu. 9th edition. Diabetes mellitus. 2019;22(1S1):1-144. (In Russ.)] doi: https://doi.org/10.14341/DM221S1

10. Yau JW, Rogers SL, Kawasaki R, et al. Global prevalence and major risk factors ofdiabetic retinopathy. Diabetes care. 2012;35(3):556-564. doi: https://doi.org/10.2337/Dc11-1909

11. Ciulla TA, Bracha P, Pollack J, Williams DF. Real-world outcomes of anti-vascular endothelial growth factor therapy in diabetic macular edema in the United States. Ophthalmol Retina. 2018;2(12):1179-1187. doi: https://doi.org/10.1016/J.Oret.2018.06.004

12. Iglicki M, Lavaque A, Ozimek $\mathrm{M}$, et al. Biomarkers and predictors for functional and anatomic outcomes for small gauge pars plana vitrectomy and peeling of the internal limiting membrane in naïve diabetic macular edema: the vital study. Plos One 2018;13(7):e0200365. doi: https://doi.org/10.1371/Journal.Pone.0200365

13. Borsey DQ, Prowse CV, Gray RS, et al. Platelet and coagulation factors in proliferative diabetic retinopathy. J Clin Pathol. 1984;37(6):659-664. doi: https://doi.org/10.1136/jcp.37.6.659

14. Madan R, Gupt B, Saluja S, et al. Coagulation profile in diabetes and its association with diabetic microvascular complications. J Assoc Physicians India. 2010;58:481-484.

15. Das R, Kerr R, Chakravarthy U, Hogg RE. Dyslipidemia and diabetic macular edema: a systematic review and meta-analysis. Ophthalmol. 2015;122(9):1820-1827. doi: https://doi.org/10.1016/J.Ophtha.2015.05.011

16. Singh RP, Habbu K, Ehlers JP, et al. The impact of systemic factors on clinical response to ranibizumab for diabetic macular edema. Ophthalmol. 2016;123(7):1581-1587. doi: https://doi.org/10.1016/J.Ophtha.2016.03.038

17. Астахов Ю.С., Залевская А.Г., Карпова И.А., и др. Факторы, влияющие на прогрессирование диабетической ретинопатии у больных сахарным диабетом 2-го типа после перевода на инсулинотерапию // РМЖ. Клиническая офтальмология. 2005. - T. 6. — №3. — C. 110-114. [Astakhov YuS, Zalevskaya AG, Karpova IA, et al. Factors, influencing diabetic retinopathy progression in type 2 diabetic patients after switching to insulin therapy. RMJ. Klinicheskaya oftal'mologiya. 2005;6(3):110-114. (In Russ.)]

18. Funatsu $H$, Yamashita $H$, Ikeda $T$, et al. Angiotensin and vascular endothelial growth factor in the vitreous fluid of patients with diabetic macular edema and other retinal disorders. Am J Ophthalmol. 2002;133(4):537-543. doi: https://doi.org/10.1016/s0002-9394(02)01323-5

19. Kerbel RS. Tumor angiogenesis. N Engl J Med. 2008;358(19):2039-2049. doi: https://doi.org/10.1056/nejmra0706596

20. Ferrara N. Role of vascular endothelial growth factor in regulation of physiological angiogenesis. Am J Physiol Cell Physiol. 2001;280(6):13581366. doi: https://doi.org/10.1152/ajpcell.2001.280.6.c1358
21. Борзилова Ю.А., Болдырева Л.А., Валеева Р.Р., и др. Уровень VEGF-а в слезной жидкости у пациентов с различными стадиями диабетической ретинопатии // Современные технологии в офтальмологии. - 2016. — №1. - C. 43-47. [Borzilova YuA, Boldyreva LA, Valeyeva RR, i dr. Uroven'VEGF-a v sleznoy zhidkosti u patsiyentov s razlichnymi stadiyami diabeticheskoy retinopatii. Sovremennyye tekhnologii v oftal'mologii. 2016;(1):43-47. (In Russ.)]

22. Homme RP, Singh M, Majumder A, et al. Remodeling of retinal architecture in diabetic retinopathy: disruption of ocular physiology and visual functions by inflammatory gene products and pyroptosis. Front Physiol. 2018;9:1268. doi: https://doi.org/10.3389/Fphys.2018.01268

23. Кузьмин А.Г., Липатов Д.В., Смирнова О.М., Шестакова М.В. АнтиVEGF препараты для лечения диабетической ретинопатии // Офтальмохирургия. - 2009. - №3. - C. 53-57. [Kuz'min AG, Lipatov DV, Smirnova OM, Shestakova MV. Anti-VEGF preparaty dlya lecheniya diabeticheskoy retinopatii. Ophthalmosurgery. 2009;(3):53-57. (In Russ.)]

24. Dugel PU, Layton A, Varma R. Diabetic macular edema diagnosis and treatment in the real world: an analysis of medicare claims data (2008 to 2010). Ophthalmic Surg Lasers Imaging Retina. 2016;47(3):258-267. doi: https://doi.org/10.3928/23258160-20160229-09

25. Funatsu $\mathrm{H}, \mathrm{Noma} H$, Mimura T, et al. Association of vitreous inflammatory factors with diabetic macular edema. Ophthalmol. 2009;1 16(1):73-79. doi: https://doi.org/10.1016/J.Ophtha.2008.09.037

26. Kwon SH, Shin JP, Kim IT, Park DH. Aqueous levels of angiopoietin-like 4 and semaphorin $3 e$ correlate with nonperfusion area and macular volume in diabetic retinopathy. Ophthalmol. 2015;122(5):968-975. doi: https://doi.org/10.1016/J.Ophtha.2015.01.007

27. Rübsam A, Parikh S, Fort PE. Role of inflammation in diabetic retinopathy. Int J Mol Sci. 2018;19(4). pii: E942. doi: https://doi.org/10.3390/ijms19040942

28. Shu DY, Lovicu FJ. Myofibroblast transdifferentiation: the dark force in ocular wound healing and fibrosis. Prog Retin Eye Res. 2017;60:44-65. doi: https://doi.org/10.1016/j.preteyeres.2017.08.001

29. Di Carlo SE, Peduto L. The perivascular origin of pathological fibroblasts. J Clin Invest. 2018;128(1):54-63. doi: https://doi.org/10.1172/JC193558

30. Arevalo JF, Maia M, Flynn HW, et al. Tractional retinal detachment following intravitreal bevacizumab (avastin) in patients with severe proliferative diabetic retinopathy. Br J Ophthalmol. 2008;92(2):213-216. doi: https://doi.org/10.1136/bjo.2007.127142

31. Pang JP, Son G, Yoon YH, et al. Combined vitrectomy with intravitreal dexamethasone implant for refractory macular edema secondary to diabetic retinopathy, retinal vein occlusion, and noninfectious posterior uveitis. Retina. 2020;40(1):56-65. doi: https://doi.org/10.1097/lae.0000000000002358

32. Choi JA, Ko SH, Park YR, et al. Retinal nerve fiber layer loss is associated with urinary albumin excretion in patients with type 2 diabetes. Ophthalmol. 2015;122(5):976-981 doi: https://doi.org/10.1016/J.Ophtha.2015.01.001

33. Wang $\mathrm{H}$, Chhablani J, Freeman WR, et al. Characterization of diabetic microaneurysms by simultaneous fluorescein angiography and spectral-domain optical coherence tomography. Am J Ophthalmol. 2012;153(5):861-867.e1. doi: https://doi.org/10.1016/j.ajo.2011.10.005

34. Early photocoagulation for diabetic retinopathy. ETDRS report number 9. Early Treatment Diabetic Retinopathy Study Research Group. Ophthalmol. 1991;98(5 Suppl):766-785. doi: https://doi.org/10.1016/s0161-6420(13)38011-7

35. Silva PS, Cavallerano JD, Haddad NM, et al. Peripheral lesions identified on ultrawide field imaging predict increased risk of diabetic retinopathy progression over 4 years. Ophthalmol. 2015;122(5):949-956. doi: https://doi.org/10.1016/J.Ophtha.2015.01.008

36. Witmer MT, Kiss S. The clinical utility of ultra-wide-field imaging [Internet]. Rev Ophthalmol. [cited 2012 March 8] Available from: https:// www.reviewofophthalmology.com/article/the-clinical-utility-of-ultrawide-field-imaging

37. Silva PS, El-Rami H, Barham R, et al. Hemorrhage and/or microaneurysm severity and count in ultrawide field images and early treatment diabetic retinopathy study photography. Ophthalmol 2017;124(7):970-976. doi: https://doi.org/10.1016/J.Ophtha.2017.02.012

38. Shah VP, Shah SA, Mrejen S, Freund KB. Subretinal hyperreflective exudation associated with neovascular agerelated macular degeneration. Retina. 2014;34(7):1281-12888 doi: https://doi.org/10.1097/IAE.0000000000000166 
39. Dugel PU, Campbell JH, Kiss S, et al. Association between early anatomic response to anti-vascular endothelial growth factor therapy and long-term outcome in diabetic macular edema. Retina. 2019;39(1):88-97. doi: https://doi.org/10.1097/lae.0000000000002110

40. Santos A, Costa M, Schwartz C, et al. Optical coherence tomography baseline predictors for initial best-corrected visual acuity response to intravitreal anti-vascular endothelial growth factor treatment in eyes with diabetic macular edema. Retina. 2018;38(6):1110-1119. doi: https://doi.org/10.1097/IAE.0000000000001687

41. Sun JK, Lin MM, Lammer J, et al. Disorganization of the retinal inner layers as a predictor of visual acuity in eyes with center-involved diabetic macular edema. JAMA Ophthalmol. 2014;132(11):1309-1316. doi: https://doi.org/10.1001/Jamaophthalmol.2014.2350

42. Sophie R, Lu N, Campochiaro PA. Predictors of functional and anatomic outcomes in patients with diabetic macular edema treated with ranibizumab. Ophthalmol. 2015;122(7):1395-1401. doi: https://doi.org/10.1016/J.Ophtha.2015.02.036

43. Santos AR, Alves D, Santos T, et al. Measurements of retinal fluid by optical coherence tomography leakage in diabetic macular edema. Retina. 2019;39(1):52-60. doi: https://doi.org/10.1097/lae.0000000000001905

44. Moein HR, Novais EA, Rebhun CB, et al. Optical coherence tomography angiography to detect macular capillary ischemia in patients with inner retinal changes after resolved diabetic macular edema. Retina. 2018;38(12):2277-2284. doi: https://doi.org/10.1097/lae.0000000000001902

45. Regatieri CV, Branchini L, Carmody J, et al. Choroidal thickness in patients with diabetic retinopathy analyzed by spectral-domain optical coherence tomography. Retina. 2012;32(3):563-568. doi: https://doi.org/10.1097/lae.0b013e31822f5678

46. Niestrata-Ortiz M, Fichna P, Stankiewicz W, Stopa M. Determining the effect of diabetes duration on retinal and choroidal thicknesses in children with type 1 diabetes mellitus. Retina. 2020;40(3):421-427. doi: https://doi.org/10.1097/lae.0000000000002420

47. Kang HM, Lee NE, Choi JH, et al. Significant reduction of both peripapillary and subfoveal choroidal thickness after panretinal photocoagulationin patients with type 2 diabetes. Retina. 2018;38(10):1905-1912. doi: https://doi.org/10.1097/lae.0000000000001804

48. Coscas G, De Benedetto U, Coscas F, et al. Hyperreflective dots: a new spectral-domain optical coherence tomography entity for follow-up and prognosis in exudative age-related macular degeneration. Ophthalmologica. 2013;229(1):32-37. doi: https://doi.org/10.1159/000342159

49. Vujosevic S, Bini S, Torresin T, et al. Hyperreflective retinal spots innormal and diabetic eyes: b-scan and en face spectral domain optical coherence tomography evaluation. Retina. 2017;37(6):1092-1103 doi: https://doi.org/10.1097/lae.0000000000001304
50. Chen $\mathrm{KC}$, Jung JJ, Curcio CA, el al. Intraretinal hyperreflective foci in acquired vilelliform lesions of the macula: clinical and histologic study. Am J Ophthalmol. 2016;164:89-98. doi: https://doi.org/10.1016/j.ajo.2016.02.002

51. Zanzottera EC, Messinger JD, Ach T, et al. The project macula retinal pigment epithelium grading system for histology and optical coherence tomography in age-related macular degeneration. Invest Ophthalmo/ Vis Sci. 2015;56(5):3253-3268. doi: https://doi.org/10.1167/iovs.15-16431

52. Pang CE, Messinger JD, Zanzottera EC, et al. The onion sign in neovascular age-related macular degeneration represents cholesterol crystals. Ophthalmol. 2015;122(11):2316-2326. doi: https://doi.org/10.1016/j.ophtha.2015.07.008

53. Sadiq MA, Soliman MK, Sarwar S, et al. Effect of vitreomacular adhesion on treatment outcomes in the ranibizumab for edema of the macula in diabetes (read-3) study. Ophthalmol. 2016;123(2):324-329. doi: https://doi.org/10.1016/J.Ophtha.2015.09.032

54. Ip MS, Domalpally A, Sun JK, Ehrlich JS. Long-term effects of therapy with ranibizumab on diabetic retinopathy severity and baseline risk factors for worsening retinopathy. Ophthalmol. 2015;122(2):367-374 doi: https://doi.org/10.1016/J.Ophtha.2014.08.048

55. Zur D, Iglicki M, Busch C, et al. Optical coherence tomography biomarkers as functional outcome predictors in diabetic macular edema treated with dexamethasone implant. Ophthalmol. 2018;125(2):267-275. doi: https://doi.org/10.1016/J.Ophtha.2017.08.031

56. Kim JH, Lee MW, Byeon SH, et al. Associations between individual retinal layer thicknesses and diabetic peripheral neuropathy using retinal layer segmentation analysis. Retina. 2018;38(11):2190-2196. doi: https://doi.org/10.1097/lae.0000000000001835

57. Ho J, Dans K, You Q, et al. Comparison of $3 \mathrm{~mm} \times 3 \mathrm{~mm}$ versus $6 \mathrm{~mm} \times 6 \mathrm{~mm}$ optical coherence tomography angiography scan sizes in the evaluation of nonproliferative diabetic retinopathy. Retina. 2019;39(2):259-264. doi: https://doi.org/10.1097/lae.0000000000001951

58. Alibhai AY, De Pretto LR, Moult EM, et al. Quantification of retinal capillary nonperfusion in diabetics using wide-field optical coherence tomography angiography. Retina. 2020;40(3):412-420. doi: https://doi.org/10.1097/lae.0000000000002403

59. Alam M, Zhang Y, Lim Jl, et al. Quantitative optical coherence tomography angiography features for objective classification and staging of diabetic retinopathy. Retina. 2018 doi: https://doi.org/10.1097/lae.0000000000002373

60. Lee J, Moon BG, Cho AR, et al. Optical coherence tomography angiography of DME and its association with anti-VEGF treatment response. Ophthalmology. 2016;123(11):2368-2375. doi: https://doi.org/10.1016/j.ophtha.2016.07.010

\section{ИНФОРМАЦИЯ ОБ АВТОРАХ [AUTHORS INFO]}

* Павлов Владислав Геннадьевич [Vladislav G. Pavlov, MD]; адрес: Россия, 119021, Москва, ул. Россолимо, д. 11, корпус A [address: 11 A Rossolimo street, 119021 Moscow, Russia]; ORCID: https://orcid.org/0000-0001-6744-4907; eLibrary SPIN: 2613-5494; e-mail: pavlovoculis@yandex.ru

Будзинская Мария Викторовна, д.м.н. [Maria V. Budzinskaya, MD, PhD]; ORCID: https://orcid.org/0000-0002-5507-8775; eLibrary SPIN: 3552-7061; e-mail: m_budzinskaya@mail.ru

Липатов Дмитрий Валентинович, д.м.н., профессор [Dmitry V. Lipatov, MD, PhD, professor]; ORCID: https://orcid.org/0000-0002-2998-3392; eLibrary SPIN: 9601-3993; e-mail: glas1966@rambler.ru

Петрачков Денис Валериевич, к.м.н. [Denis V. Petrachkov, MD, PhD];

ORCID: https://orcid.org/0000-0003-4757-5584; eLibrary SPIN: 7736-1801; e-mail: petrachkov@doctor.com

\section{ЦИТИРОВАТЬ:}

Будзинская М.В., Липатов Д.В, Павлов В.Г., Петрачков Д.В. Биомаркеры при диабетической ретинопатии // Сахарный диабет. - 2020. - Т. 23. — №1. - C. 88-94. doi: 10.14341/DM10045

\section{TO CITE THIS ARTICLE:}

Budzinskaya MV, Lipatov DV, Pavlov VG, Petrachkov DV. Biomarkers for diabetic retinopathy. Diabetes Mellitus. 2020;23(1):88-94. doi: 10.14341/DM10045 